\title{
NON-CONTACT AUXILIARY FIXTURE FOR BETTER MACHINING OF THIN FLEXIBLE WORKPIECE USING EDDY CURRENTS
}

\author{
Robinderpal Singh \\ Advanced Manufacturing Lab (AML) \\ School of Engineering \\ University of Guelph \\ Guelph, Ontario, Canada. \\ robinder@uoguelph.ca
}

\author{
Ibrahim M. Deiab \\ Advanced Manufacturing Lab (AML) \\ School of Engineering \\ University of Guelph \\ Guelph, Ontario, Canada. \\ ideiab@uoguelph.ca
}

\begin{abstract}
Thin flexible parts are widely used in the aerospace industry and require a more precise surface finish. The main problem in achieving high-quality product is the vibration induced during machining of thin-walled parts. Clamping these thin parts or using rigid supports for parts, such as for propeller blades, lead to distortion. This issue may be solved using a noncontact auxiliary fixture. The new fixture developed in this research is an eddy current damper for passive control of vibrations in electrically conducting workpieces, using neodymium permanent magnets without any external power source. Two thin aluminum beams with different cross-section and same length were used to test the developed fixture. To increase the overall damping, conducting sheets were attached at the free end of beams. Impact and machining tests were conducted to validate the new fixture. The results showed higher damping and better machining stability leading to enhanced surface quality of the workpiece.
\end{abstract}

Keywords- Thin-flexible parts; vibration; fixture; eddy current damper; passive damping

\section{INTRODUCTION}

Machining of thin-walled workpieces is required in several industries, most notably the aerospace industry. During machining of thin parts, vibration is the main factor affecting the surface finish and tolerance. Milling is one of the most common processes used for thin workpieces. To improve the stability of the workpiece, i.e. to damp the vibrations, various methods are applied including choosing optimized machining parameters or using primary or auxiliary contacting or non-contacting fixtures [1]. In recent studies, distinct theoretical models have been created, and systems have been analyzed for damping of vibration, mainly in the cantilever beams. This research analyzes and controls the vibrations during machining of cantilever beamlike workpieces for better stability and surface finish.

In the aerospace industry, accuracy and precision of the machining process and the manufactured parts, along with the production rate and the tool life, are the primary areas for research. The parts that are more flexible, such as, the impellers, propeller blades etc., need much higher accuracy and a smooth surface finish. Chatter is widely studied in the literature. Lai et al. [2] studied chatter characteristics of a cylindrical thin-walled workpiece. The relation of the wall thickness and the inner diameter was analyzed to study machining vibration characteristics. Numerical and experimental methods were used to investigate the dynamic characteristics of the workpiece. Campa et al. [3] came up with a method to damp the vibrations in thin-floor milling using a bull-nose end mill. A mathematical model was created to predict the behavior of workpiece during the milling process and to generate a stability lobe diagram for selecting the optimal spindle speed for better stability during machining. Junjin et al. [4] worked on the use of magnetorheological (MR) fluid, which can be tuned using a magnetic field by changing its state from liquid to solid by aligning the magnetic particles in a single direction, to damp the vibrations in a thin cantilever beam and found a considerable change in the vibrations.

\section{EDDY CURRENT DAMPER}

Damping of vibrations in thin-walled electrically conducting workpieces can be achieved using magnetism. The use of eddy currents to oppose the motion of the workpiece utilizes the basic principle of using a magnetic field for vibration damping.

When a conductor moves in a magnetic field, electrical currents are induced in it, known as eddy currents. These currents generate a counter magnetic field with an opposing force, known as the Lorentz force. This Lorentz force (F) is directly opposite to the direction of movement of the conductor moving with a velocity (v) with respect to the magnetic field (B), as shown in Figure 1. When the movement of the conductor is perpendicular to the polar axis of the magnet, the radial component of magnetic field does not contribute to the damping effect due to lack of much interaction with the conductor. On the other hand, when the conductor moves parallel to the axis of poles of magnet, only the radial component of magnetic flux is responsible for inducing eddy currents and creating damping forces. 


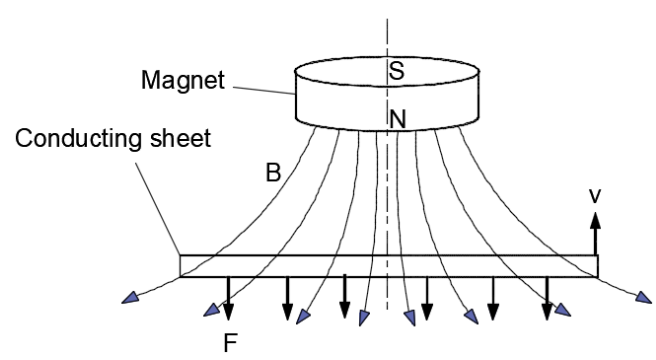

(a)

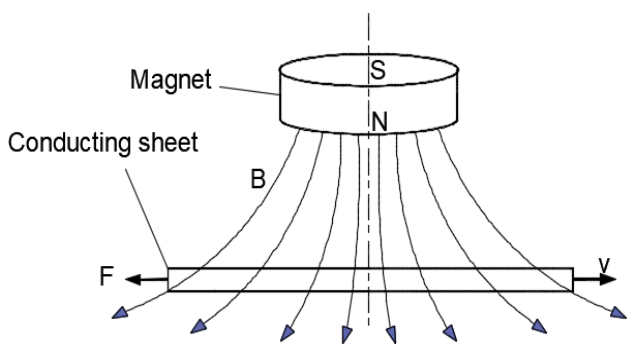

(b)

Figure 1: (a) Damping force using radial magnetic flux, and (b) Damping using axial magnetic flux

The induced current density $(\vec{J})$ can be obtained as,

$$
\vec{J}=\sigma(\vec{v} \times \vec{B})
$$

In the above equation, the conductor has conductivity $\sigma$, velocity $\vec{v}$ w.r.t the magnetic field and magnetic flux density $\vec{B}$.

The damping force of the system due to the magnetic field can be determined analytically. The damping force depends on the magnetic flux density $(B)$, the relative velocity of the vibrations $(v)$, and the properties of the conductor used, which includes a thickness $(\delta)$ and conductivity $(\sigma)$.

From the relation given, the damping force due to axial magnetic field lines, as used by Bae et al., [5], is given as

$$
F=-\left(\sigma \delta B^{2} S\left(\alpha_{1}+\alpha_{2}\right)\right) v
$$

Where $S$ is the area of pole projection, and $\alpha_{1}$ and $\alpha_{2}$ are the conducting sheet's effect on damping force.

The case of a radial magnetic flux, as studied by Sodano et al. [6] is given as:

$$
F=\int_{v} J \times B d v=-2 k \pi \sigma \delta \int_{0}^{r_{c}} y B_{y}^{2}\left(y, l_{g}\right) d y
$$

With $\mathrm{k}$ as the spring coefficient of the system, $r_{c}$ as the equivalent radius of conducting sheet, and $l_{g}$ as the air gap between the magnet and the conducting sheet.

This eddy current creates an opposing field and force which opposes the motion of the conductor. This damping effect can be used for a thin-walled cantilever beam using a strong magnetic field around it such that the oscillation of the beam in the magnetic field induces a Lorentz force or damping force. For better results and more control, electromagnets can be used in this vibration damping methods [7]. The eddy current damping process is contact-less, wear-free, fast and safe. Also, with the electromagnets, the magnetic field or the force can be controlled using different input current or coil turns, making it more adjustable for different work conditions [8].

Sodano with Jae-Sung Bae [6] developed a passive eddy current damper which used radial components of magnetic flux for vibration damping, using permanent magnets. The damper showed an increase in damping ratio up to 150 times for a thin cantilever beam, when fixed at the free end. The above study was continued by Sodano et al. [9] to change the passive current damper to an active eddy current damper by using electromagnets instead of permanent magnets. With this active system, the magnetic field strength can be varied actively so as to induce eddy currents even without much movement of the conductor. This system is effective when the movement of the conductor in a magnetic field is not enough to induce significant eddy currents.

Another study was performed by Cheng et al. [10] using a permanent magnet for damping of vibrations. In their work, a conduction coil was attached to the cantilever beam to vary the resistance during induction of eddy currents using a permanent magnet. Ebrahimi et al. [11] worked on passive eddy current damper and developed an analytical model to select the permanent magnets of optimum strength and size.

\section{EXPERIMENTAL SETUP AND METHODOLOGY}

This research was conducted to develop a non-contacting auxiliary fixture, as shown in Figure 2, for machining stability of cantilever-like workpieces, such as propeller and impeller blades, to suppress machining vibrations and enhance the surface finish of the end-product. The fixture developed has an aluminum frame to limit interaction with the magnetic field. The 'magnet holder' holds the neodymium magnet and can be moved to change the gap between the magnet and the workpiece. Allen bolts are used to lock the 'magnet holders' at any point. Use of permanent magnets on either side of the workpiece is an ideal option when the workpiece moves perpendicular to the polaraxis of the magnets for maximum interaction of magnetic field lines.

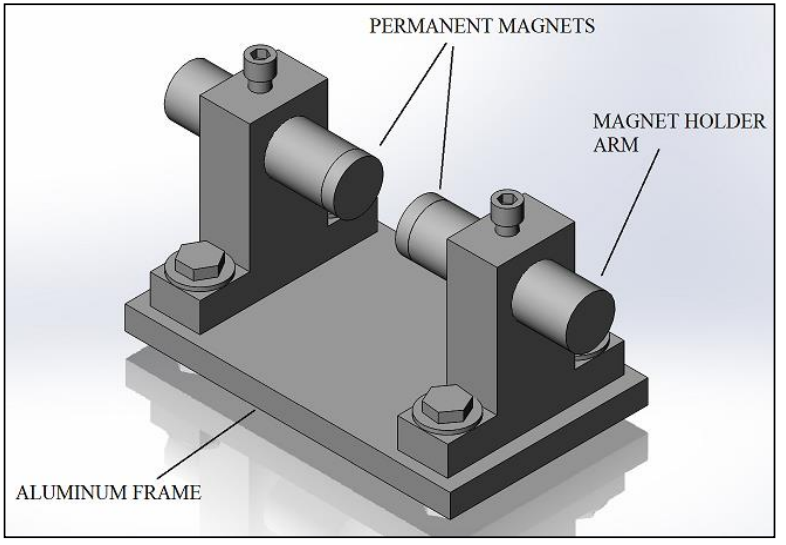

Figure 2: Developed experimental setup 
Two Aluminum beams (6061-T6), one of dimensions 170 $\mathrm{mm} \times 25 \mathrm{~mm} \times 6 \mathrm{~mm}$ (referred as 'thick beam') and the other of dimensions $170 \mathrm{~mm}$ x $38.5 \mathrm{~mm}$ x $3.58 \mathrm{~mm}$ (referred as 'thin beam'), were selected as the workpiece because of resemblance to a propeller blade. Permanent magnets (Neodymium N-48), of diameter $25.4 \mathrm{~mm}$ and thickness $6.35 \mathrm{~mm}$ each, were used to induce the eddy currents in the workpiece to produce the damping effect whenever there is a motion in the workpiece due to induced vibrations. The beams were secured on one end in a vise and the eddy current fixture was placed at the free end, as shown in Figure 3. Aluminum sheets $(50 \mathrm{~mm} \times 37 \mathrm{~mm}$ x 1.5 $\mathrm{mm}$ ) were glued at the free ends of both beams (as in Figure 3(b) and 3(c)).

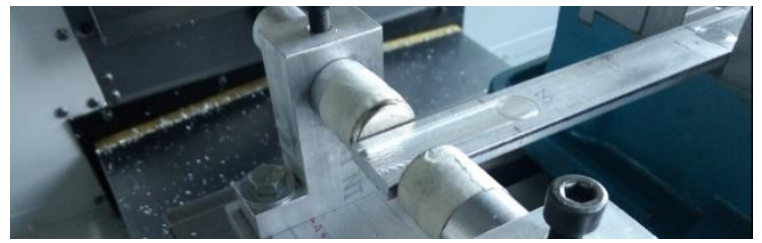

(a)

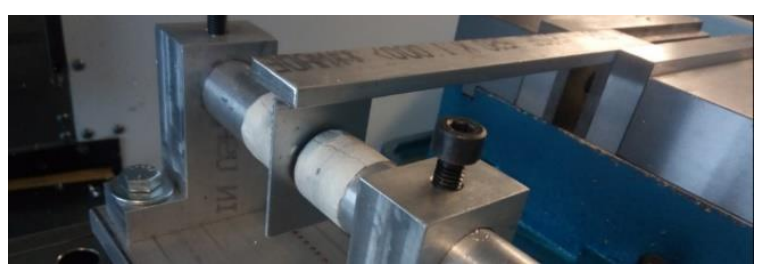

(b)

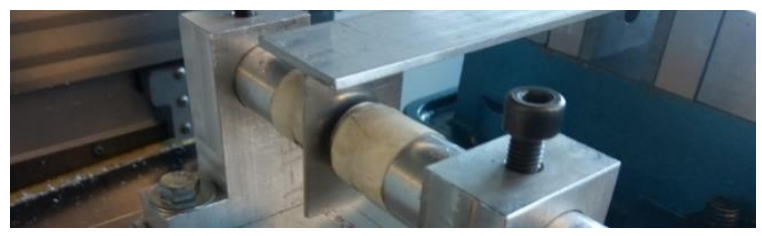

(c)

Figure 3. Different setups of the test workpiece (a) Beam 1 (without additional conducting element), (b) Beam 2 (thick beam with aluminum sheet), and (c) Beam 3 (thin beam with aluminum sheet element)

The three beam arrangements (Beam-1 i.e. thick beam without the additional aluminum sheet, Beam-2 i.e. thick beam with the aluminum sheet glued and Beam-3 i.e. thin beam with the aluminum sheet glued) used are given in Figure 3, with a constant gap of $0.8 \mathrm{~mm}$ between the magnet and the beam for each arrangement. The natural frequencies and the modes of each beam, with and without the fixture, were obtained using impact hammer test through LMS Test.lab by Siemens (as shown in Figure 4). The frequency response functions were then obtained from the LMS Test-lab software to get the average damping ratios and damping ratios for each mode for all the beams. The results of Test-lab, for natural frequencies and modes, were confirmed using ANSYS modal analysis. ANSYS Maxwell was used to simulate the magnets to see the maximum magnetic flux on the beam.

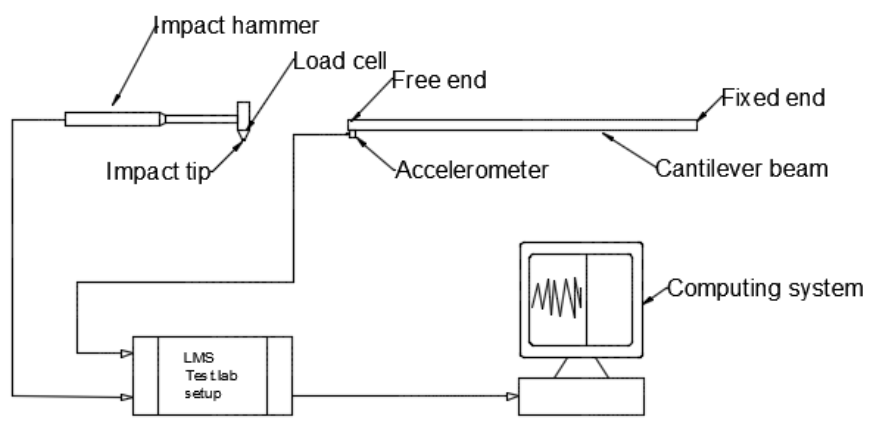

Figure 4: Impact test setup schematic

Observing the damping effect, Beam-2 and Beam-3 were machined in HAAS 3 -axis milling machine at $2400 \mathrm{rpm}$ of spindle speed with a feed rate of 15 inches per minute to measure the acceleration during machining and to observe the surface finish of the machined workpiece. A 4 flute 3/8-inch HSS flat end mill tool was used for machining of the workpiece to create a 3-inch slot of 20 thou depth from the free end inwards, at the center of beams with aluminum sheets attached. Siemens Test.lab 'Signature' module was used to measure the online acceleration signals. A profilometer was used to measure the surface roughness of the workpiece.

\section{RESULTS AND DISCUSSION}

The permanent magnets were simulated using the ANSYS Maxwell software and it was observed that the magnetic flux density increases as the distance between the magnets decreases. Thus, as per the magnetic flux density results, the air gap between the cantilever beam and the magnets on either side was kept constant at $0.8 \mathrm{~mm}$. The damping effect can be clearly seen in the frequency response function plots in Figure 5.

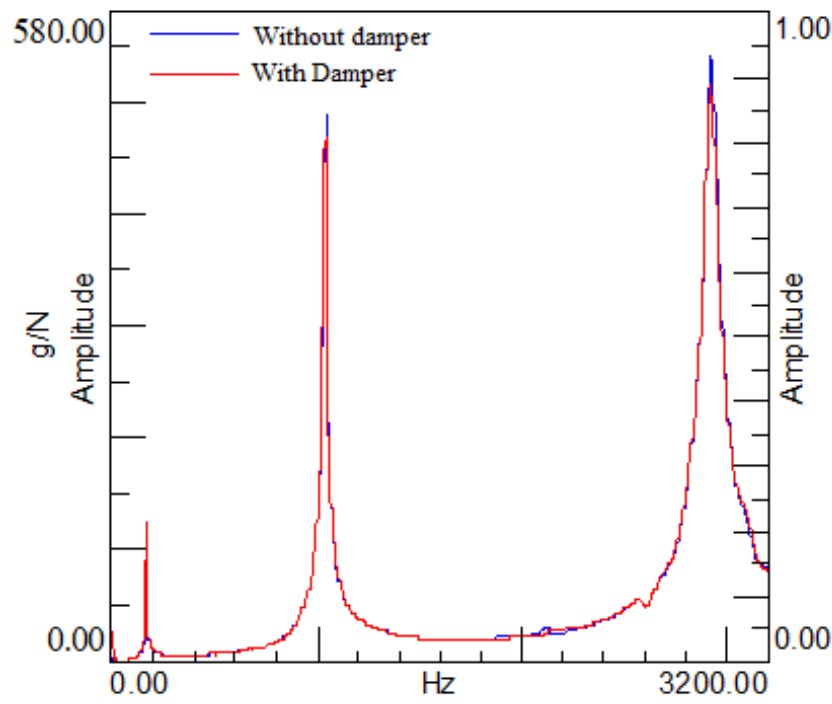

(a) 


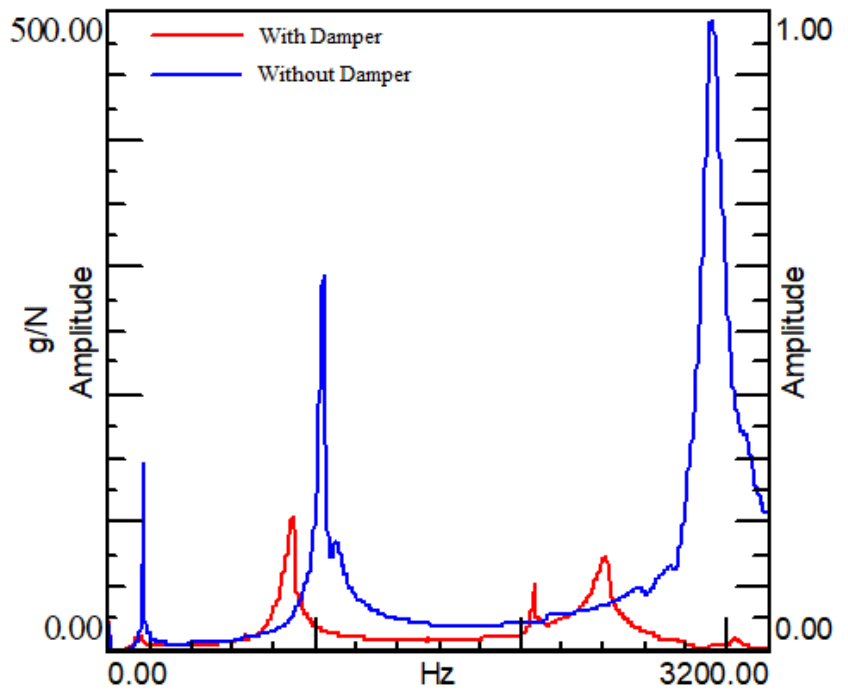

(b)

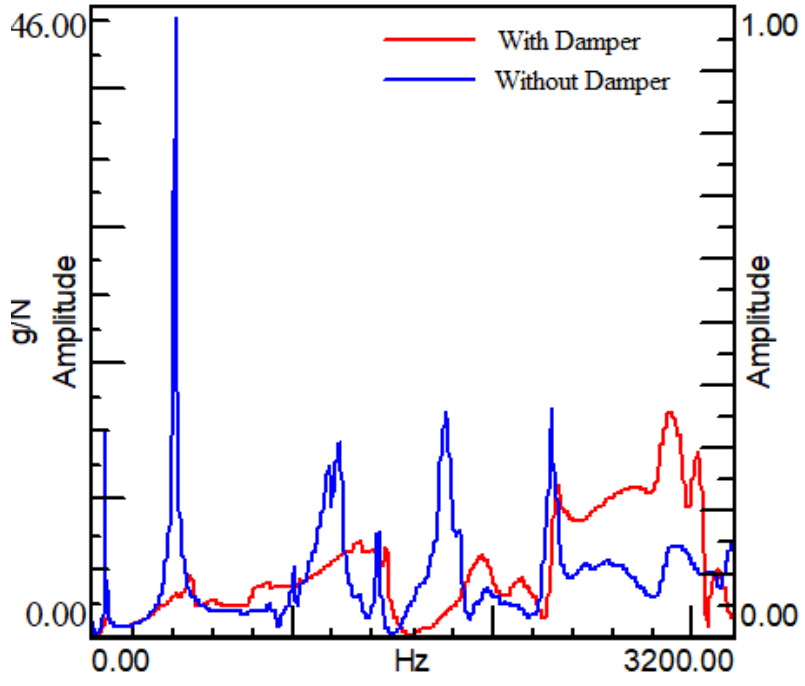

(c)

Figure 5: Frequency response function plots for (a) Beam-1, (b) Beam-2, and (c) Beam-3.

\section{IV.I. MODAL ANALYSIS}

The frequency response function (FRF) plots for the three cases of the beams (with and without aluminum sheet) were obtained from LMS Test.lab software, given in Figure 5. For Beam-1, as from the FRF plot in Figure 5(a), there is not much effect of the damper on the amplitude and damping ratio by the use of damper.

The average damping ratio ( $\%$ ) for Beam-1 increased from $2.95 \%$ to $3.41 \%$ with the use of damper. However, for Beam-2, it changed from $3.51 \%$ to $12.26 \%$ and for Beam-3, it moved from $6.66 \%$ to $40.38 \%$. Table-1 and Table- 2 shows the change in natural frequency, amplitude and damping ratio for the first three modes of Beam-2 and Beam-3, which were considered for this research. The maximum damping ratio and minimum amplitude for Beam-2 and Beam-3 were observed at mode 1 of each beam arrangement where the damping ratio increased more than 7 times for the Beam-2 and 12 times for Beam-3 and the amplitude decreased by more than 10 times, when the damper was placed at the free end of the beams.

The Beam-2 and Beam-3, with a conducting sheet attached to the free end, experienced the maximum damping because of use of the axial component of the magnetic field and the closeproximity of the magnets, providing a higher magnetic flux for more eddy currents. The closely packed magnetic field lines passing through the beam had the maximum interaction with the beam, even with minimum movements. The thicker beam with conducting sheet has higher stiffness making it less affected by the eddy currents than the thin beam. The damping force also depends on the thickness of the conducting sheet attached. Making the sheet thicker will increase the eddy current's density but can also make the system unstable.

TABLE 1: Modal testing results for Beam-2

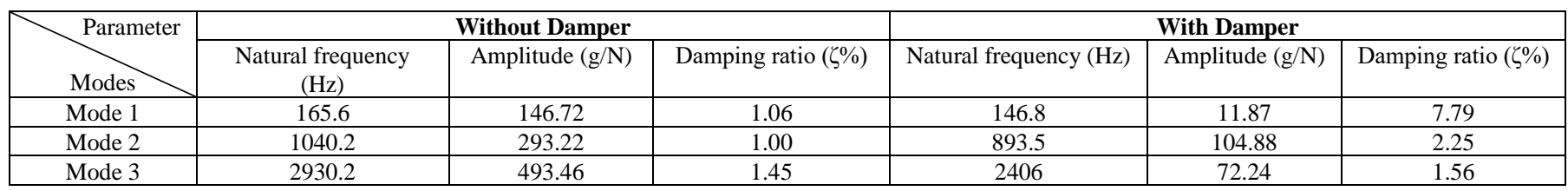

TABLE 2: Modal testing results for Beam-3

\begin{tabular}{|c|c|c|c|c|c|c|}
\hline Parameter & \multicolumn{3}{|c|}{ Without Damper } & \multicolumn{3}{|c|}{ With Damper } \\
\hline Modes & $\begin{array}{c}\text { Natural frequency } \\
(\mathrm{Hz})\end{array}$ & Amplitude $(\mathrm{g} / \mathrm{N})$ & Damping ratio $(\zeta \%)$ & Natural frequency $(\mathrm{Hz})$ & Amplitude (g/N) & Damping ratio ( $\zeta \%)$ \\
\hline Mode 1 & 68.7 & 15.27 & 1.73 & 71.8 & 1.47 & 21.53 \\
\hline
\end{tabular}




\section{IV.II. MACHINING TEST}

The machining tests were performed for Beam-2 and Beam3 only, because of the significant damping observed during modal analysis. The machining tests showed a reduction in the acceleration of both the beams during machining. For Beam-2, the maximum positive acceleration reduced from 415 to $180 \mathrm{~g}$. For thin beam with aluminum sheet, the maximum acceleration dropped from $368 \mathrm{~g}$ to $164 \mathrm{~g}$, as shown in the Figure 6. This change in the acceleration can be related to the damping shown in the modal testing. However, Beam-3 has lower stiffness which causes it to bend a bit when the cutting tool makes a contact, making the beam in less contact with the tool, as can be seen in the machined surface without the damping fixture. This causes undercutting of the beam. When the damping fixture was applied at the end of the beam, the damping force resisted the motion of the beam, making a better contact with the cutting tool. Also, the irregular pattern of the acceleration was caused by continuously changing of overhung mass when the cutting tool moves toward the clamped end of the beam.
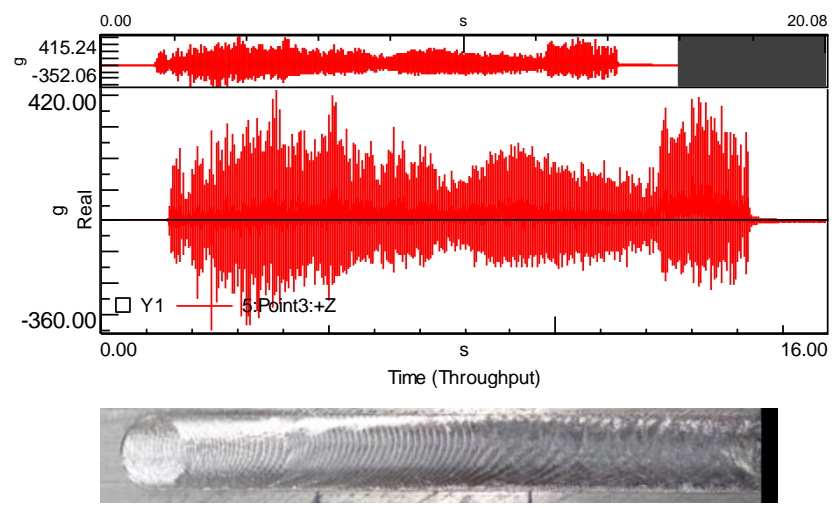

(a)
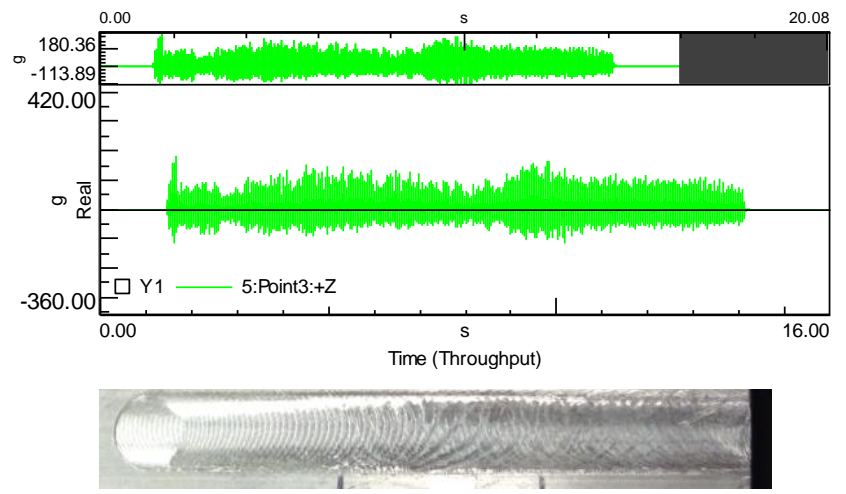

(b)

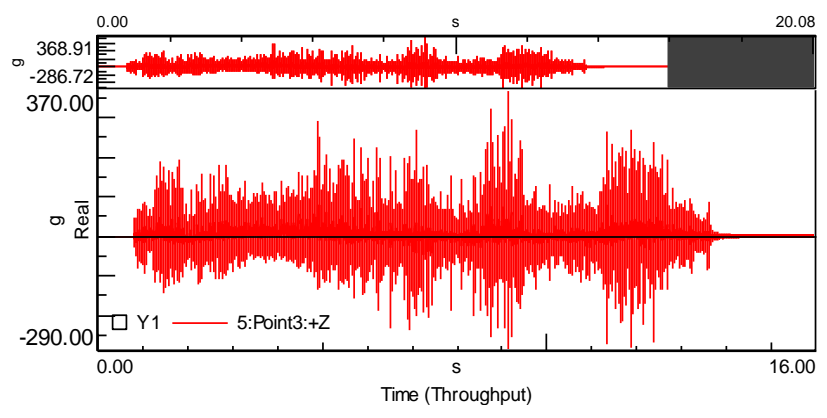

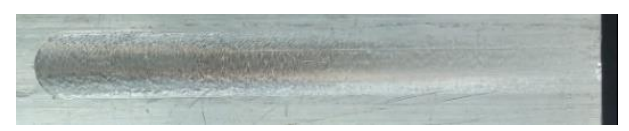

(c)

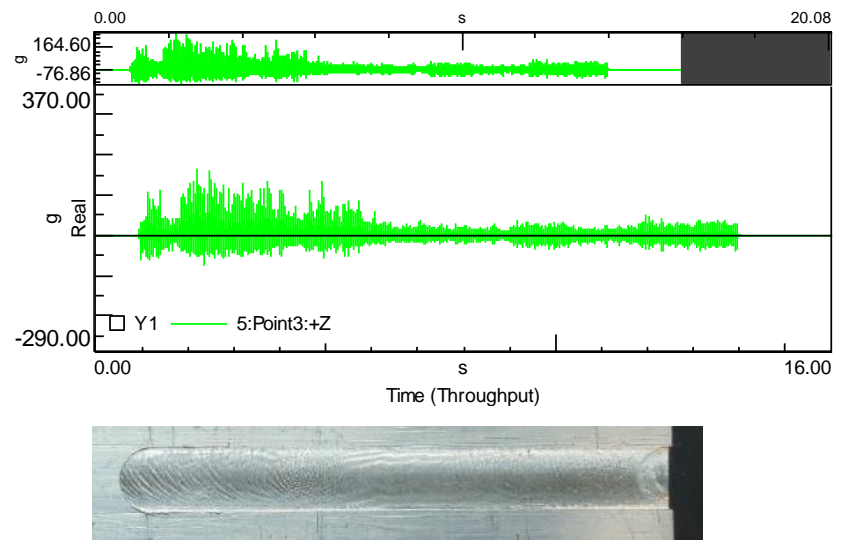

(d)

Figure 6: Machining results at 2400 rpm spindle speed, 15 inches per minute of feed and 20 thousandth-of-an-inch cutting depth for (a) Beam-2 without damper, (b) Beam-2 with damper, (c) Beam-3 without damper, and (d) Beam-3 with damper.

The average surface roughness of the beams was measured using a profilometer and Beam-2 showed a better surface finish, with average roughness values $\left(R_{a}\right)$ changing from $3.52 \mu \mathrm{m}$ to $2.17 \mu \mathrm{m}$, while for Beam-3 the initial impact causes deflection of the beam making it difficult to compare the surface roughness, but a higher material removal rate was observed (undercutting was prevented by the damper).

\section{CONCLUSIONS}

Machining of thin-walled parts is considered a challenge because of the chatter induced due to lower stiffness of workpieces. To counter this issue, an auxiliary fixture, based on eddy currents, was developed to damp the vibrations in a cantilever beam and to improve the surface finish of the product. The fixture developed is a passive eddy current damper which is simple to use and does not need a power supply for its operation.

The damping force (Lorentz force) applied by the eddy current damper reduced the vibrations in the workpiece when there is an interaction of a conductor with the magnetic field. The damping force was increased by attaching a conducting sheet to the workpiece to increase the magnetic flux density with respect to the conductor sheet. The damping force was observed as maximum when the cantilever beam workpiece has low stiffness, a conducting sheet is attached to the free end and the gap between the magnets is kept small. The fixture proposed highly damps the first three modes of the tested beams.

The modal testing and the machining tests validated the usability of eddy current dampers for better machining stability, by suppressing chatter. Better surface finish and high material removal rate was observed by using the proposed auxiliary fixture. Future work will focus on optimizing the thickness of the conducting sheet attached and also on using it as a tuned mass damper. 


\section{ACKNOWNLEDGEMENT}

This work was supported by the Natural Sciences and Engineering Research Council of Canada (NSERC).

\section{REFERENCES}

[1] G. Quintana and J. Ciurana, "Chatter in machining processes: A review," Int. J. Mach. Tools Manuf., vol. 51, no. 5, pp. 363-376, 2011

[2] J. Y. Chang, G. J. Lai, and M. F. Chen, "A study on the chatter characteristics of the thin wall cylindrical workpiece," Int. J. Mach. Tools Manuf., vol. 34, no. 4, pp. 489-498, 1994.

[3] F. J. Campa, L. N. Lopez De Lacalle, and A. Celaya, "Chatter avoidance in the milling of thin floors with bull-nose end mills: Model and stability diagrams," Int. J. Mach. Tools Manuf., vol. 51, no. 1, pp. 43-53, 2011.

[4] J. Ma, D. Zhang, B. Wu, M. Luo, and B. Chen, "Vibration suppression of thin-walled workpiece machining considering external damping properties based on magnetorheological fluids flexible fixture," Chinese J. Aeronaut., vol. 29, no. 4, pp. 1074-1083, 2016.

[5] D. Bae, Jae-Sung;kwak,Moon k.;Inman, "Vibration suppression of a cantilever beam using eddy current damper," J. Sound Vib., vol. 284, pp. 805-824, 2004.

[6] H. A. Sodano, J. S. Bae, D. J. Inman, and W. Keith Belvin, "Concept and model of eddy current damper for vibration suppression of a beam," J. Sound Vib., vol. 288, no. 4-5, pp. 1177-1196, 2005.

[7] H. D. Wiederick, N. Gauthier, D. A. Campbell, and P. Rochon, "Magnetic braking: Simple theory and experiment," Am. J. Phys., vol. 55, no. 6, pp. 500-503, 1987.

[8] O. Rodrigues, O. Taskar, S. Sawardekar, H. Clemente, and G. Dalvi, "Design \& Fabrication of Eddy Current Braking System," Int. Res. J. Eng. Technol., vol. 3, no. 4, pp. 809-815, 2016.

[9] H. A. Sodano and D. J. Inman, "Non-contact vibration control system employing an active eddy current damper," J. Sound Vib., vol. 305, no. 4-5, pp. 596-613, 2007.

[10] T. Cheng and I. Oh, "Vibration Suppression of Flexible Beam Using Electromagnetic Shunt Damper," Ieee Trans. Magn., vol. 45, no. 6, pp. 2758-2761, 2009.

[11] B. Ebrahimi, M. B. Khamesee, and F. Golnaraghi, "Permanent magnet configuration in design of an eddy current damper," Microsyst. Technol., vol. 16, no. 1-2, pp. 19-24, 2010. 